\title{
Symmetry and asymmetry in bacterial and organellae genomes
}

\author{
Michael Sadovsky \\ ICM SB RAS, Krasnoyarsk, Russia \\ msad@icm.krasn.ru
}

\author{
Maria Senashova \\ ICM SB RAS, Krasnoyarsk, Russia \\ msen@icm.krasn.ru
}

\begin{abstract}
Basic mirror symmetry is found, for all three types of genomes (bacterial, chloroplasts and mitochondria). The patterns are arranged by the formally identified fragments of a genome, of the same length converted into triplet frequency dictionaries. Prevalent pattern for all genome types looks like a three ray star with the fragments gathered into the clusters. Some deviations and violations of the typical pattern are observed and discussed.
\end{abstract}

Keywords - nucleotide sequence, triplet, frequency, clustering, order, GC-content

Motivation and aim

Motivation

It is well-known that various fragments of a genome differ each other in their role in inherited information processing. We investigated the reciprocal problem of the impact of those functional difference onto the structurally determined difference of the formally identified fragments of a genome. We concentrated on single chromosome genomes (bacteria, chloroplasts and mitochondria).

Aim

The aim of this work is to compare the patterns revealed through the distribution of formally identified fragments of a genome into the triplet frequency space, in terms of the symmetry (or asymmetry) of that former.

\section{Methods}

We used two-step transformation of a genome to reveal the inner structuredness. Firstly, each genome was covered with a set of (overlapping) tiles. Each tile is a subsequence of the given length $L$; they located along a genome with the step $T$. We used $L=603$ and $T=11$; it is important that the length $L$ is odd and divisible by 3 , and the step $T$ is indivisible by 3 . Each tile is labeled with so called relative phase index. The index marks up the tiles in terms their functional role to be found within a genome, according to an annotation.

To determine the index, we traced the location of the central nucleotide at the tile. If the central nucleotide falls within a non-coding region, it takes index value $j$. When the central nucleotide falls within a coding region (regardless the inner exon-intron structure of a gene), then the index value is 0 , if the remainder of the division of the distance (defined in nucleotides number) from the start position of a coding region to the central nucleotide of the tile position by 3 . Correspondingly, the index value is 1 and 2 , if the remainder is equal to 1 or 2 . These phase indices are determined for the leading strand. Reciprocally, the indices take values $j,-0$

,-1 or -2 , for the genes located at the ladder strand. Obviously, the remainder and the distance to the central nucleotide of the tile must determined from the "end" of the annotated fragment, since any gene bank stores a singly strand, only.
As soon, as each tile is labeled, it must be converted into a triplet frequency dictionary $W_{I}(n)$, where $l$ is the index value, and $n$ is the location of the tile along a genome. Hence, a genome is transformed into an ensemble of the points in 63dimensional metric space with Euclidean metrics; we eliminated a triplet from the analysis, since the sum of all 64 frequencies yields 1 . It should be stressed that the frequency dictionary contains the triplets counted with the reading frame shift equal to 3 ; in other words, the count of the triplets corresponds to the translation reading frame shift; such design revealed the difference between the coding and non-coding regions, as well as the genes located in leading and ladder strands.

Practically, the triplet with the least standard deviation determined over the entire ensemble was excluded and the motivation is apparent: it contributes least of all into separability of the points. These were the triplets GGG for bacteria, GCG for chloroplasts and ACT for mitochondria. Also, for each tile GC-content has been calculated, as well as the genome-wide one.

Next, we studied the inner structure of the ensemble, through the analysis of the distribution of those points in the metric space provided by frequencies, and principal components [1]. We used freely distributed software VidaExperts [2, 3].

The inner structuredness has been studied for a family of a single chromosome genomes, among them are bacteria genomes (168), chloroplast genomes (789) and mitochondria genomes, both animal (463) and plant (45).

\section{Results}

Examination of structure produced by the distribution of the tiles converted into triplet frequency dictionary exhibits four-cluster pattern for greater part of genomes. This is a starlike pattern with three rays comprising the tiles of the specific phase indices. Remarkable fact is that outer parts (rays) of the star are comprised with the tiles laid at the coding regions; the inner cluster comprises the tiles belonging to non-coding regions of a genome. The interlocation of the tiles follows the symmetry composition: the first (core) ray comprises the tiles of 2 and -2 indices; two other rays incorporate the tiles of 0 and -1 , and -0 and 1 phase indices. The central core of the pattern is occupied with $j$ phase tiles.

The most common fact is that two sets of clusters corresponding to three indices and determined over the leading strand and the ladder one, reciprocally, mirror each other. Mirroring here means that the phase index runs the circuit over the cluster clockwise, for leading strand, and counter clockwise, for ladder strand.

This pattern is absolutely typical for chloroplast genomes: no exclusions have been found in the analysis of 798 entities. It should be noticed that chloroplast genomes indeed have some extra cluster; that latter comprises the tiles covering the area of very densely located tRNA genes in a genome. These 
tiles have extremely high local GC-content, thus gathering into an individual cluster. The profiles of GC-content along chloroplast genomes could be seen in [4].
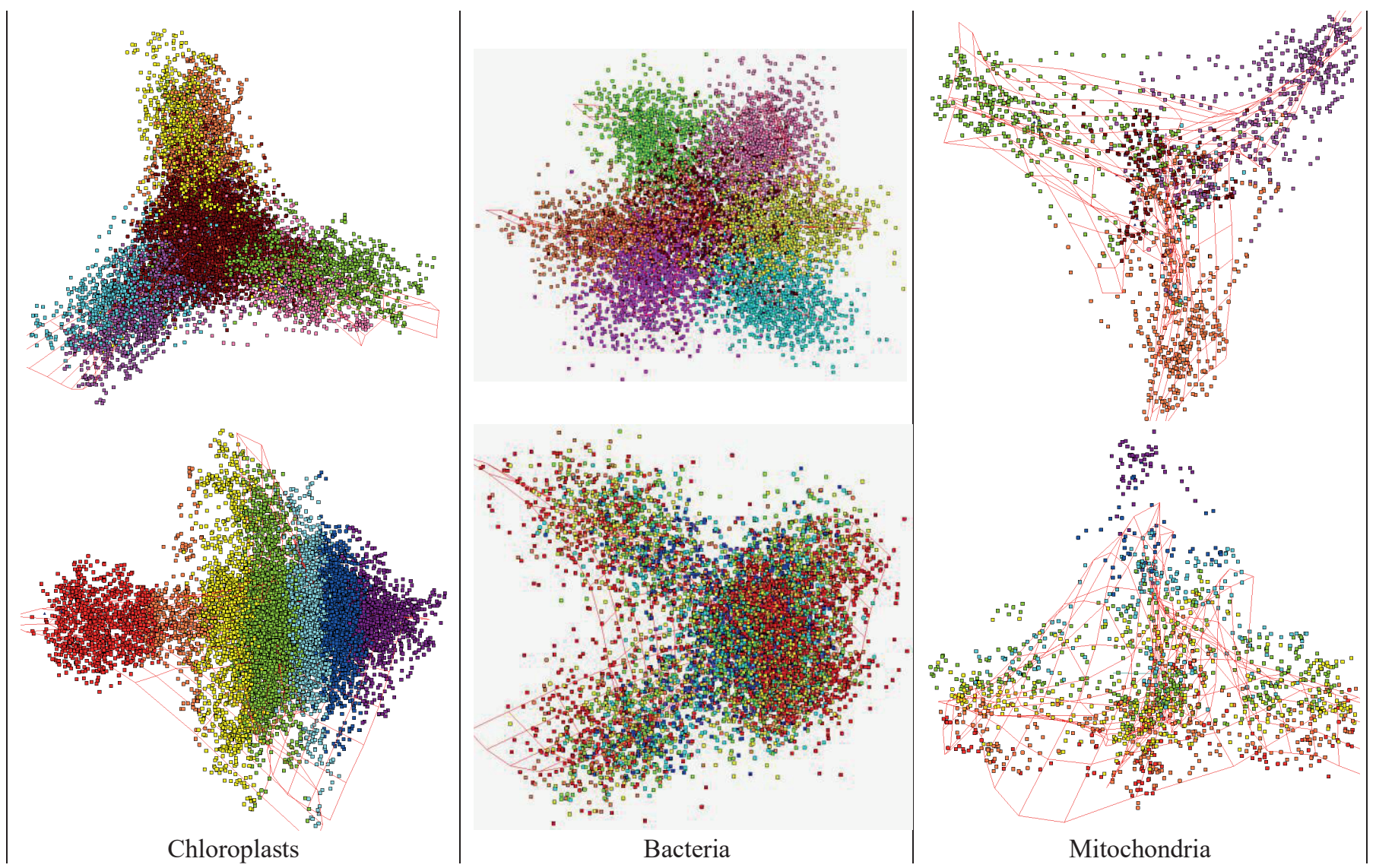

Fig. 1. Typical patterns observed for three types of genomes, see explanations in text.

Bacterial genomes exhibit diverse variety of patterns. Meanwhile, it should be said that three-ray pattern is typical for GC-reach and GC-poor bacterial genomes. Middle level GC-content bacterial genomes may have various patterns, among them is that one shown in Fig.1. Greater majority of genomes shows the three-ray star-like pattern, with the tiles falling into non-coding regions located in the center of the pattern, and the rays comprising the tiles indexed as described above.

The most unexpected and significantly less structured patterns exhibit mitochondrial genomes. To begin with, there is tremendous difference between animal and plant mitochondrial genomes, in terms of the clustering.

Fig.1. shows the examples of the patterns to be observed for the genomes of various origin. Upper row of figures

\section{References}

[1] K. Fukunaga, Introduction to Statistical Pattern Recognition (2 Edition), (1990) Academic Press, 593 p., DOI: 10.1016/B978-0-08047865-4.50017-X

[2] Gorban, A., Popova, T., \& Zinovyev, A. (2005). Codon usage trajectories and 7-cluster structure of 143 complete bacterial genomic sequences. Physica A: Statistical Mechanics and its Applications, 353, 365-387. presents the clustering observed in the projection of the second and the third principal components. Coloring in these pictures corresponds to the relative index phases: $j$ is brown, 0 and -0 are blue and pink, 1 and -1 are green and violet, 2 and -2 are orange and yellow. For any genome with mirror symmetry the phases 2 and -2 always form a cluster; for other patterns there might be some other combinations.

Lower row in Fig.1 shows GC-content distribution, over the pattern. That latter is shown in the first and the second principal component projection. Coloring represents the local values of GC-content of each tile, changing in rainbow order from red (the greatest $\mathrm{GC}$-content) to violet (the lowest $\mathrm{GC}$ content).

[3] Gorban, A. N., \& Zinovyev, A. Y. (2008). Elastic maps and nets for approximating principal manifolds and their application to microarray data visualization. In Principal manifolds for data visualization and dimension reduction (pp. 96-130). Springer, Berlin, Heidelberg.

[4] Sadovsky M.G., Putintseva Yu. A., Senashova M. Yu. (2018) Eight Clusters, Synchrony of Evolution and Unique Symmetry in Chloroplast Genomes: The Offering from Triplets // In: Chloroplasts and Cytoplasm: Structure and Functions (ser. Cell Biology Research Progress), Eds. Carl Dejesus and Lourdes Trask, Nova Science Publishers, Inc., N.-Y., pp.25-95. 